\title{
Calculation of hardening process parameters for locomotive parts
}

\author{
Alexander Buynosov ${ }^{1, *}$, Vasily Lapshin ${ }^{1}$, Alexander Smolyaninov ${ }^{1}$, and Albert Dinislamov ${ }^{1}$ \\ ${ }^{1}$ Ural State University of Railway Transport, 620034 Yekaterinburg, Russia
}

\begin{abstract}
The existing theoretical models of heating by concentrated sources with a high energy density generally describe processes with deep penetration welding of part surfaces. The purpose of this study is to identify the factors that have a major effect on hardening of parts through heat treatment with a high-speed or pulsed scanning stationary heat source, which creates a uniform temperature field. Using methods of regression analysis, the authors derived equations for calculating the hardening depth in the proposed hardening process, the rate and time of steel cooling in a critical temperature range. The paper presents the calculated parameters of the hardening process in which parts, including wheel flanges of locomotives, are heated by a plasma arc in nitrogen. The findings can be used to reduce costs of complex experiments aimed at selecting surface heat hardening parameters to increase the service life of locomotive mechanical parts.
\end{abstract}

\section{Introduction}

Different types of hardening are used to improve the reliability of steel parts throughout the entire life cycle. At present, surface hardening of steels by powerful concentrated energy sources, such as a laser beam and plasma arc, have been widely adopted. In order to avoid surface melting and increasing the area to be treated, heat source scanning is used. A plasma arc with transverse electromagnetic oscillations is widely used to harden various mechanical parts of locomotives made of carbon steels, including wheel flanges [1].

A review of foreign [1-8] and Russian [9-12] studies shows that heating parameters for concentrated sources with a high energy density are chosen on the basis of deep penetration welding procedures [2-4] or determined empirically [5]. After a part is hardened, a hardness profile of the hardened layer is measured using a destructive method. The procedure is repeated until the required depth and hardness of the thermally hardened layer are achieved. This requires considerable time and money. The most common techniques include low-temperature plasma nitriding $[2,3]$ and plasma heat treatment $[4,11]$. The key factors influencing hardening of steel parts, however, have not yet been established.

The purpose of this study is to identify the factors that have a major effect on hardening a part through heat treatment with a high-speed or pulsed scanning stationary heat source.

\footnotetext{
*Corresponding author: byinosov@mail.ru
} 
The scientific novelty of the paper is a method designed to calculate steel hardening parameters for locomotive parts through heat treatment with a high-speed or pulsed scanning stationary heat source, which creates a uniform temperature field.

\section{Selection of a calculation methodology}

To calculate parameters of temperature fields, we can use an equation [4] that describes heating of a semi-infinite body for an instantaneous source:

$$
T=\frac{Q}{c \gamma \cdot \sqrt{a \pi t}} \cdot e^{\frac{z^{2}}{4 a t}},
$$

where $T$ is the temperature; $Q$ is the energy density; $c$ is the specific heat capacity; $\gamma$ is the density of steel; $a$ is the coefficient of thermal diffusivity; $t$ is the time of heat propagation; $z$ is the depth from the body surface.

When different mechanical parts of the locomotive are hardened with a plasma $\operatorname{arc}(K=$ $1.4 \mathrm{~cm} / \mathrm{s} ; \delta=2.8 \mathrm{~cm} ; a=0.08 \mathrm{~cm}^{2} / \mathrm{s}$ [10]), the conditions $K \cdot \delta / 4 a=12.25$ is satisfied. Therefore, the solution to the equation (1) can be used. The same solution is applicable to deep layers. For example, the thickness of a hardened layer $(2 \mathrm{~mm})$ for wheelsets is small compared to the thickness of a flange (maximum: 33, minimum: $25-28 \mathrm{~mm}$ ).

Define a heat flux as $q=\eta \cdot N / S$ and the arc time as $t_{0}$, where $\eta$ is the efficiency of a heat source; $N$ is the power of a heat source; $S$ is the area of heat treatment. For a heat source moving at a speed $V, N=U \cdot I$, ( $U$ is the voltage, $I$ is the arc current), $S=b \cdot d$ ( $b$ is the oscillation width of the arc, $d$ is the effective arc diameter, $t_{0}=d / V$ ).

During the operation of the plasma arc that generates the heat flux $q$, at a time point $t$, for the period $d t^{\prime}$, the energy $d Q=q \cdot d t^{\prime}$ will be emitted (Fig. 1).

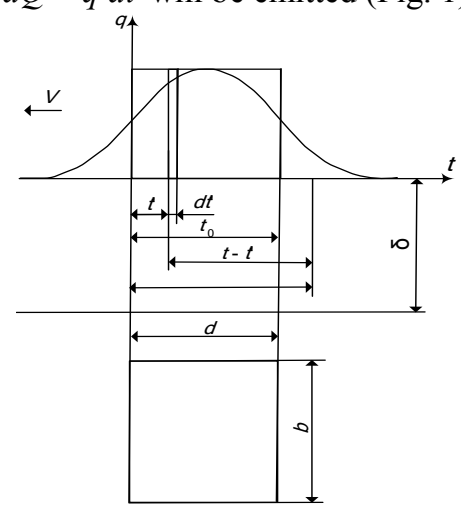

Fig. 1. Diagram of heating by a normally distributed source reduced to a uniformly distributed source.

By substituting $d Q$ into (1), we obtain an equation for temperature change

$$
T-T_{0}=\frac{q}{c \gamma \sqrt{\pi a}} \int_{0}^{B} \frac{d t^{\prime}}{\sqrt{t-t^{\prime}}} \cdot e^{-\frac{z^{2}}{4 a \cdot\left(t-t^{\prime}\right)}},
$$

where $B=t$ at $t<t_{0}$ and $B=t_{0}$ at $t \geq t_{0} ; T_{0}$ is the initial temperature; $t^{\prime}$ is the heating time.

It is clear that the maximum temperature $\left(T_{m}\right)$ is reached on the surface (at $z=0$ ) at the end of heat treatment $t=t_{0}$. We can easily obtain it by integrating (2): 


$$
T_{m}-T_{0}=\frac{2 q}{c \gamma} \cdot \sqrt{\frac{t_{0}}{\pi a}} .
$$

Let us introduce relative parameters: time $\tau=t / t_{0}$; operating time of the source $\tau^{\prime}=t^{\prime} / t_{0}$; depth $Z=z /\left(4<a \cdot t_{0}\right)^{1 / 2}$; temperature $\theta=\left(T-T_{0}\right) /\left(T_{m}-T_{0}\right)$. The expression (2) can be written as:

$$
\theta=\frac{1}{2} \int_{0}^{B} \frac{d \tau^{\prime}}{\sqrt{\tau-\tau^{\prime}}} \cdot \mathrm{e}^{-\frac{Z^{2}}{\tau-\tau^{\prime}}},
$$

where $B=\tau$ at $\tau<1$ and $B=1$ at $\tau \geq 1$.

For the surface $Z=0$, the integral (4) can be finally expressed as:

$$
\begin{aligned}
& \theta=\sqrt{\tau}-\sqrt{\tau-1} \\
& \theta=\sqrt{\tau} \text { for } \tau<1 .
\end{aligned}
$$

The expressions (4), (5) and (6) have the same form for stationary pulsed and highspeed sources.

\section{Calculation of heat hardening parameters}

It is reasonable to use numerical methods for calculating thermal conductivity parameters. Results of calculations with the equations (4), (5) and (6) are presented in Figure 2.

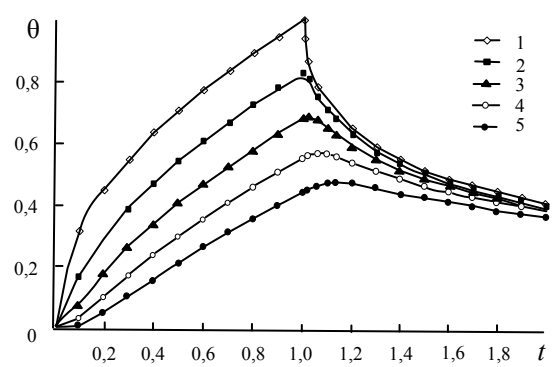

Fig. 2. Dependence of the relative temperature $(\tau)$ on the relative temperature $(\theta)$ for different depths: $1-\mathrm{Z}=0 ; 2-\mathrm{Z}=0.1 ; 3-\mathrm{Z}=0.2 ; 4-\mathrm{Z}=0.3 ; 5-\mathrm{Z}=0.4$.

For a moving heat source, it is more convenient to count the time from the end of the exposure to the source (the surface temperature is maximal). The relative temperature in a new coordinate system can be written as:

$$
\theta=\frac{1}{2} \int_{0}^{1} \frac{d t^{\prime}}{\sqrt{\tau+\tau^{\prime}}} \cdot e^{-\frac{Z^{2}}{\tau+\tau^{\prime}}}
$$

for points at a certain depth of the part (body), and

$$
\theta=\sqrt{\tau+1}-\sqrt{\tau}
$$




$$
\tau=\frac{1}{4}\left(\frac{1}{\theta}-\theta\right)^{2}
$$

for points on the surface of the part (body).

The residence time of metal in austenite state $t_{a}$ is very important in the heat hardening process for locomotive mechanical parts [12]:

$$
\tau_{a}=\frac{t_{a}}{t_{0}}=\frac{1}{4} \cdot\left(2+\frac{1}{\theta_{a}^{2}}-3 \cdot \theta_{a}^{2}\right) .
$$

In the equation (10), the relative temperature $\theta=\left(T_{a}-T_{0}\right) /\left(T_{m}-T_{0}\right)$.

When the maximum temperature $\theta_{m}$ is reached at a depth $Z$, in order to determine the relative time $\tau_{m}$, it is necessary that $d \theta / d \tau=0$, then:

$$
\tau_{m}=2 \cdot Z^{2}-\frac{\int_{0}^{1} \frac{\tau^{\prime} d \tau^{\prime}}{\sqrt{\left(\tau_{m}+\tau^{\prime}\right)^{5}}} \cdot e^{-\frac{Z^{2}}{\tau_{m}+\tau^{\prime}}}}{\int_{0}^{1} \frac{d \tau^{\prime}}{\sqrt{\left(\tau_{m}+\tau^{\prime}\right)^{5}}} \cdot e^{-\frac{Z^{2}}{\tau_{m}+\tau^{\prime}}}} .
$$

By substituting (11) into (7), we can determine $\theta_{m}$. The relative parameters calculated with the equations (7) and (11) are given in Table 1 . In this case, the hardening depth can be limited to $Z=0.4$.

Table 1. Values of relative parameters $Z, \tau_{m}$ and $\theta_{m}$.

\begin{tabular}{|c|c|c|}
\hline$Z$ & $\tau_{m}$ & $\theta_{m}$ \\
\hline 0 & 0 & 1 \\
\hline 0.05 & 0.0006857 & 0.914149 \\
\hline 0.10 & 0.0035267 & 0.834054 \\
\hline 0.20 & 0.0199325 & 0.691799 \\
\hline 0.30 & 0.0588132 & 0.573651 \\
\hline 0.40 & 0.1313577 & 0.478503 \\
\hline
\end{tabular}

Figures $3 \mathrm{a}$ and $3 \mathrm{~b}$ show dependences $\tau_{m}(Z)$ and $\theta_{m}(Z)$, respectively. For the data given in Table 1 , these dependences can be approximated by nonlinear functions $\tau_{m}=1.235 \cdot Z^{2.522}$ and $\theta_{m}=1-1.2089 \cdot Z^{0,8729}$, respectively.
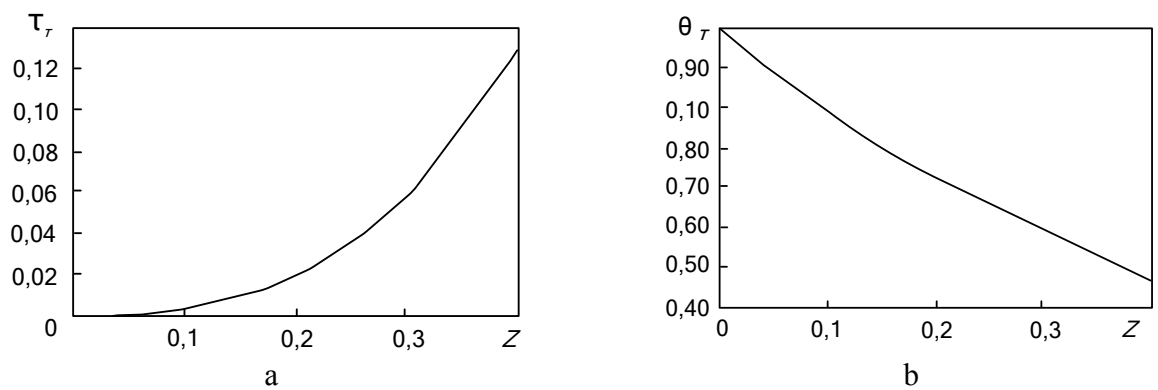

Fig. 3. Dependence on the relative depth $Z$ for: a - relative time $\tau_{m}$; $\mathrm{b}$ - relative maximum temperature $\theta_{m}$. 
The relative time $\tau_{m}$ and maximum relative heating depth $Z_{m}$ calculated at $T_{0}=950{ }^{\circ} \mathrm{C}$ and $T_{m}=1,000-1,500{ }^{\circ} \mathrm{C}$ are given in Table 2 .

Table 2. Values of temperature $T_{m}$, and relative parameters $Z, \tau_{m}$ and $\theta_{m}$.

\begin{tabular}{|c|c|c|c|}
\hline$T_{m},{ }^{\circ} \mathrm{C}$ & $\theta_{m}=950 / T_{m}$ & $\tau_{m}$ & $Z_{m}$ \\
\hline 1,000 & 0.950000 & 0.00019995 & 0.0287207 \\
\hline 1,100 & 0.863636 & 0.00213545 & 0.0810998 \\
\hline 1,200 & 0.791666 & 0.00645315 & 0.1280839 \\
\hline 1,300 & 0.730769 & 0.01329445 & 0.1708493 \\
\hline 1,400 & 0.678571 & 0.02271107 & 0.2102661 \\
\hline 1,500 & 0.633330 & 0.03473166 & 0.2469703 \\
\hline 1,600 & 0.593750 & 0.49409390 & 0.2814682 \\
\hline
\end{tabular}

Figures $4 \mathrm{a}$ and $4 \mathrm{~b}$ present dependences $\tau_{m}\left(T_{m}\right)$ and $Z_{m}\left(T_{m}\right)$, respectively.
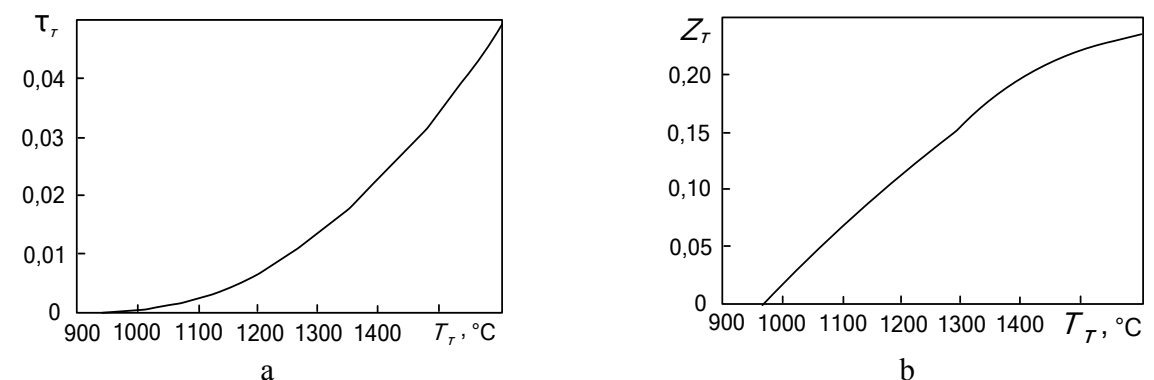

Fig. 4. Dependence on the maximum surface heating temperature $T_{m}$ for: a - relative time $\tau_{m} ; \mathrm{b}-$ relative depth of the hardened layer $Z_{m}$.

As it can be seen from Table 2 and Figure 4b, when the heating surface temperature of the part falls from 1,500 to $1,000{ }^{\circ} \mathrm{C}$, the heating depth decreases tenfold. Therefore, maintaining the required surface temperature is the key factor for high-quality hardening of locomotive mechanical parts.

Based on the calculated values given in Tables 1 and 2, an equation for calculating the hardening depth was derived using regression analysis $[5,13]$.

$$
Z_{m}=0,7838 \cdot(1-\theta)^{1,1485} \text {. }
$$

When using the approximate equation (12) in calculations, the maximum relative error is equal to 0.003 in the most common depth range $Z=0.1-0.3$.

When analyzing the hardening process for a band of grade 2 in [14], the authors of the study [15] consider the cooling time $t_{8 / 5}$ in a temperature range of 800 to $500{ }^{\circ} \mathrm{C}$ and the band cooling rate in a temperature range of 600 to $500{ }^{\circ} \mathrm{C}$. The relative cooling time in the body (at a depth) can be expressed by the relation $\tau_{8 / 5}=\tau_{500}-\tau_{800}$. Relative times $\tau_{500}, \tau_{550}$ and $\tau_{800}$ are calculated by the formulas (9) and (10). The relative cooling rate of the part in the body can be defined as:

$$
W_{550}=\frac{d \theta}{d \tau}=\frac{1}{4} \cdot \int_{0}^{1} \frac{\left[2 Z^{2}-\left(\tau_{550}+\tau^{\prime}\right)\right] d \tau^{\prime}}{\sqrt{\left(\tau_{550}+\tau^{\prime}\right)^{5}}} \cdot e^{-\frac{Z^{2}}{\tau_{550}+\tau^{\prime}}} .
$$


The relative values of time $\tau_{8 / 5}$ and rate $W_{550}$, determined as a function of the maximum surface heating temperature $T_{m}$ at $T_{a}=950{ }^{\circ} \mathrm{C}$ and $T_{0}=0{ }^{\circ} \mathrm{C}$, are given in Table 3 and Figure 5.

Table 3. Relative time and rate as a function of the maximum surface heating temperature.

\begin{tabular}{|c|c|c|c|c|c|c|c|}
\hline \multirow{2}{*}{$T,{ }^{\circ} \mathrm{C}$} & \multirow{2}{*}{$\tau_{500}$} & \multirow{2}{*}{$\tau_{550}$} & \multirow{2}{*}{$\tau_{800}$} & \multicolumn{2}{|c|}{ in the body } & \multicolumn{2}{c|}{ on the surface } \\
\cline { 5 - 8 } & & & $\tau_{8 / 5}$ & $W_{550}$ & $\tau_{8 / 5}$ & $W_{550}$ \\
\hline 1,000 & 0.5608 & 0.4004 & 0.049 & 0.5118 & 0.3663 & 0.5119 & 0.3663 \\
\hline 1,100 & 0.7484 & 0.5492 & 0.0913 & 0.6571 & 0.2666 & 0.6568 & 0.2667 \\
\hline 1,200 & 0.9502 & 0.7093 & 0.1389 & 0.8113 & 0.2014 & 0.8098 & 0.2015 \\
\hline 1,300 & 1.1675 & 0.8817 & 0.1918 & 0.9757 & 0.1563 & 0.9722 & 0.1565 \\
\hline 1,400 & 1.4013 & 1.0672 & 0.2500 & 1.5371 & 0.1240 & 1.1446 & 0.1242 \\
\hline 1,500 & 1.6521 & 1.2665 & 0.3138 & 1.3383 & 0.1001 & 1.3278 & 0.1004 \\
\hline 1,600 & 1.9205 & 1.4799 & 0.3834 & 1.5371 & 0.0821 & 1.5219 & 0.0824 \\
\hline
\end{tabular}

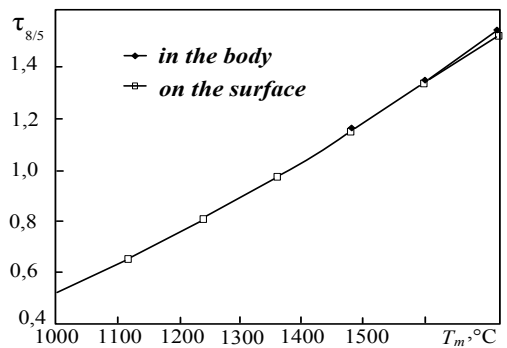

a

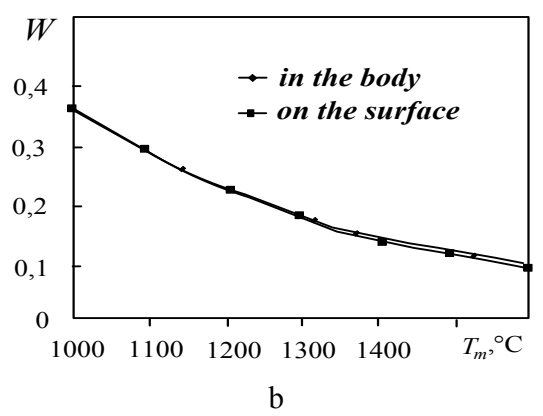

Fig. 5. Dependence on the maximum surface heating temperature $T_{m}$ for: a - relative cooling time $\tau_{8 / 5}$ in a temperature range $800-500{ }^{\circ} \mathrm{C} ; \mathrm{b}$ - relative cooling rate $W$ at $550{ }^{\circ} \mathrm{C}$.

As it can be seen from Figure 5, the dependences in the body and on the surface are almost identical. This means that various layers of the part follow a similar cooling pattern at different heating temperatures. Hence, hardening of the part through heat treatment with a high-speed or pulsed scanning stationary heat source is mainly determined by two factors: the maximum temperature and the surface heating time.

Since the heating time is defined as a ratio of the diameter of the heat source to the speed of the source itself, the following parameters should be maintained constant to ensure the high-quality hardening process: 1) arc current and voltage that affect the effective diameter of the heat source; 2) constant speed of the source.

\section{Conclusion}

As a result of the studies, the key factors that influence hardening of steel locomotive parts were determined. Mathematical expressions were obtained for calculating the hardening depth, cooling time and rate of steels in a critical temperature range generated by a scanning stationary heat source, which creates a uniform temperature field. These findings should be compared with experimental data, results of computer-aided 3D modeling of dynamics and kinematics of the hardening process, and other hardening methods (e.g., electric arc). 


\section{References}

1. S. Radchenko, D. Dorokhov, I. Gryadunov, Journal of Chemical Technology and Metallurgy, J. E 1, 50, 104112 (2015).

2. T. Aizawa, K. Wasa, Micromachines, J. E 8, 157 (2017).

3. V.A. Korotkov, M.V. Zlokazov, Journal of Friction and Wear, J. E 2, 133136 (2014).

4. M. Zhang, C. Chen, X. Wang, J. Hou, S. Zhang, G. Zhu, H. Jing, Acta Metallurgica Sinica (English Letters), J. E 3, 27, 521529 (2014).

5. V. Ivantsivsky, K. Parts, V. Popov, Applied Mechanics and Materials, J. E 788 (2015).

6. V.I. Pyndak, A.E. Novikov, Metal Science and Heat Treatment, J. E 3-4, 58, 226230 (2016).

7. G.M. Zhuravlev, A.E. Gvozdev, A.E. Cheglov, N.N. Sergeev, O.M. Gubanov, Steel in Translation, J. E 6, 47, 399411 (2017).

8. A.S. Kolpakov, N.I. Kardonina, Thermal Engineering, J. E 2, 63, 150155 (2016).

9. D.N. Trushnikov, D.S. Belinin, Y.D. Shitsyn, Modern problems of science and education, 2, 95 (2014). (in Russian)

10. S.A. Mikaeva, A.S. Mikaeva, M.S. Rodyukov, Uprochnjajushhie tehnologii i pokrytija [Strengthening Technologies and Coatings], 3, 128-131 (2018). (in Russian)

11. S.Yu. Petrov, R.V. Kokorin, S.M Baban, Tekhnologiya Mashinostroeniya [Technology of Mechanical Engineering], 4, 37-44 (2017). (in Russian)

12. V.V. Muravyev, S.Yu. Petrov, L.V. Volkova, Defektoskopija [Russian Journal of Nondestructive Testing], 10, 64-74 (2015). (in Russian)

13. O.V. Pilipenko, S.J. Radchenko, D.O. Dorokhov, I.M. Gryadunov, International Journal of Applied Engineering Research, J. E 13 (2017).

14. GOST 398-2010. Rough tyres for railway rolling stock. Specifications (Standartinform, Moscow, 2011). (in Russian)

15. V.V. Girzhon, A.V. Ovchinnikov, Metal Science and Heat Treatment, J. E 11-12, 58, 719723 (2017). 